E-033 OUTCOMES OF INTRACRANIAL CAROTID ARTERY ANEURYSMS TREATED WITH NOVEL SECOND GENERATION COILS: RESULTS FROM THE SMART REGISTRY

E Almallouhi* ${ }^{*}$ M Anadani, S Al kasab, M Sattur, A Spiotta. Medical University of South Carolina, Charleston, SC

10.1136/neurintsurg-2019-SNIS.108

Background Flow diversion is a viable treatment modality for the embolization of proximal intracranial carotid artery aneurysms (cavernous, superior hypophyseal, ophthalmic). In this study, we evaluated the outcomes of patients with internal carotid artery (ICA) aneurysms who were treated with using novel second generation coils in the SMART registry.

Methods We reviewed the multicenter, prospective SMART registry that enrolled patients who underwent endovascular treatment using Penumbra SMART ${ }^{\mathrm{TM}}$ Coils. Study endpoints include peri-procedural device-related serious adverse events, the occlusion status immediately post procedure as well as a follow-up angiogram after one year. We included only patients who underwent coiling for intracranial carotid aneurysms in this analysis.

Results Out of 906 patients included in the SMART registry, $131(14.5 \%)$ patients were treated for cavernous, ophthalmic, or superior hypophyseal artery aneurysms (age $56.7 \pm 12.7 \mathrm{yr}$, $88.5 \%$ were females, and $7.6 \%$ were ruptured). Most of the aneurysms were wide-necked (defined as an aneurysm with a dome-to-neck ratio less than 2.0 and/or a neck length of 4 $\mathrm{mm}$ or more) (86 patients, $65.6 \%)$. Sixty patients $(45.8 \%)$ underwent stent-assisted coiling and 21 patients (16\%) underwent balloon-assisted coiling. Device related serious adverse effects were noticed in one patient $(0.8 \%)$ and periprocedural mortality occurred in one patient $(0.8 \%)$. Immediate Raymond class I was achieved in 37 patients (28.2\%) and one-year Raymond class I was achieved in $56 / 79$ patients (70.9\%). Oneyear recanalization occurred in 8 patients $(6.1 \%)$.

Conclusions In this large, contemporary multicenter registry of patients, coil embolization using the SAMRT coils offers a reasonably safe and effective treatment of intracranial ICA aneurysms, as demonstrated by the high rates of complete one-year aneurysm occlusion and the low rate of recanalization and complications.

Disclosures E. Almallouhi: None. M. Anadani: None. S. Al kasab: None. M. Sattur: None. A. Spiotta: 2; C; Penumbra, Cerenovus, Minnetronix.

\section{E-034 OPTIMAL MANAGEMENT APPROACH FOR TREATING ANTERIOR COMMUNICATING ARTERY ANEURYSM}

A Sweid*, N Herial, S Tjoumakaris, S Rahm, C Andrews, M Gooch, R Rosenwasser, P Jabbour. Neurosurgery, Thomas Jefferson University, Philadelphia, PA

\subsection{6/neurintsurg-2019-SNIS.109}

Introduction Coiling has been the first line armamentarium for treating intracranial aneurysms, especially ruptured ones. Coiling has a relatively high recurrence rate as compared to clipping which leads to increased costs and procedural risks.

Purpose We aimed to determine complication rates, occlusion outcomes, functional status, and mortality of Anterior communicating artery (ACOM) aneurysms following coiling. We also determined re-treatment rate and compared occlusion outcomes between recoiling and clipping of recurrences. We also assessed factors predictive of aneurysms recurrence.

Materials and methods We performed a retrospective cohort study of consecutive patients with ACOM aneurysms.

Results 516 ACOM aneurysms were included (Av age 55.5 years, $54.3 \%$ female). $78 \%$ (401) presented as ruptured aneurysms, $55.6 \%$ presented with a high-grade $\mathrm{H} \& \mathrm{H}$. The average aneurysm size was $5.8 \mathrm{~mm}(\mathrm{SD}=2.8)$. 93\%(478) were treated with coiling as stand-alone divided into primary coiling $86 \%$ (412), balloon-assisted coiling 12\%(57), and stent-assisted coiling $2 \%(9)$. While $7 \%(37)$ were treated with coiling in addition to clipping, coiling was placed to secure the dome, and then the aneurysm was clipped during the same hospital stay in acute settings. At the end of the procedure, $\geq 95 \%$ occlusion was achieved in $90.9 \%$ of the cases. The average length of hospital stay was 15.8 days, and the mortality rate was $7.2 \%$. There was a significant six folds higher aneurysm recurrence in the coiled group reaching $17.3 \%(78)$ while in the clipped/coiled group the recurrence rate was $2.9 \%(1)$ $(p=0.026)$. Of all patients with recurrence $(n=79), 28$ were treated with clipping, 22 with coiling, 16 with coiling followed by clipping due to recurrence, and 13 patients were not re-treated. Out of the whole 38 patients who received coiling at least once, 34 needed recoiling once, 3 required recoiling twice, and 1 patient required recoiling four times. Approximately $76 \%$ of coiled patients $(n=29)$ showed recurrence, while none of the clipped patients showed recurrence $(p<0.01)$. There was no significant difference in the proportion of complications between the two groups (25\% vs.18\%, $\mathrm{p}=0.56)$. Complete occlusion was achieved in $65 \%(\mathrm{n}=52)$ on latest follow up. All patients who were clipped showed complete occlusion, while $32 \%$ of the coiled patients showed complete occlusion $(\mathrm{p}<0.01)$. RRS class II was seen in $18.9 \%$ $(n=15)$, class IIIa in $6 \%(n=5)$, and class IIIb in $2.5 \%(n=2)$. There was no significant difference in the mean mRS score between the both groups $(p=0.11)$. The average duration of follow up following retreatment was 10 months(95\%CI:4-15). Most patients had an mRS score $\leq 2(n=60)$. Variables that are significantly associated with aneurysm recurrence are increased aneurysm size and ruptured presentation.

Conclusion The recurrence rate of coiled aneurysms compared to clipping was associated with 6 folds higher rate of recurrence. Clipping of a recurred ACOM aneurysms achieves higher occlusion outcomes, lower recurrence rate, and comparable risk of complication and functional outcome compared to coiling. Increasing aneurysm size and ruptured status was a significant predictor of aneurysm recurrence.

Disclosures A. Sweid: None. N. Herial: None. S. Tjoumakaris: None. S. Rahm: None. C. Andrews: None. M. Gooch: None. R. Rosenwasser: None. P. Jabbour: None.

\section{E-035 TREATMENT OUTCOMES OF UNRUPTURED CEREBRAL ANEURYSMS IN SEPTUAGENARIANS AND OCTOGENARIANS- A SINGLE CENTER EXPERIENCE}

G Kaur*, J Cooper, L Melathe, F Al-Mufti, C Gandhi, J Santarelli. Neurosurgery, Westchester Medical Center, Valhalla, NY

\subsection{6/neurintsurg-2019-SNIS. 110}

Introduction Elective aneurysm treatment in elderly patients may be associated with a higher risk of complications due to frailty, atherosclerosis, and tortuosity. Because of these 